\title{
PROXIMINALITY IN GEODESIC SPACES
}

\author{
A. KAEWCHAROEN AND W. A. KIRK \\ Received 4 May 2006; Revised 1 August 2006; Accepted 10 August 2006
}

Let $X$ be a complete CAT( 0$)$ space with the geodesic extension property and Alexandrov curvature bounded below. It is shown that if $C$ is a closed subset of $X$, then the set of points of $X$ which have a unique nearest point in $C$ is $G_{\delta}$ and of the second Baire category in $X$. If, in addition, $C$ is bounded, then the set of points of $X$ which have a unique farthest point in $C$ is dense in $X$. A proximity result for set-valued mappings is also included.

Copyright (C) 2006 A. Kaewcharoen and W. A. Kirk. This is an open access article distributed under the Creative Commons Attribution License, which permits unrestricted use, distribution, and reproduction in any medium, provided the original work is properly cited.

\section{Introduction}

This paper is primarily motivated by a recent paper of Zamfirescu [17], in which it is shown that for any compact set $C$ in a complete length space $X$ without bifurcating geodesics, the nearest point projection $P_{C}$ of $X$ onto $C$ is properly single valued at most points of $X$, that is, on a set of second Baire category. We show here that the same is true for a closed subset of a complete $\mathrm{CAT}(0)$ space $X$, provided $X$ has the geodesic extension property and has Alexandrov curvature bounded below. We also show that if $C$ is bounded and closed, then the set of points of $X$ which have a unique farthest point in $C$ is dense in $X$. These are extensions pioneering results, see Edelstein [7] and Stečkin [16]. Also see [15] for other generic results.

A metric space is a CAT(0) space (the term is due to Gromov—see, e.g., [1, page 159]) if it is geodesically connected, and if every geodesic triangle in $X$ is at least as "thin" as its comparison triangle in the Euclidean plane. Precise definitions are given below. For a detailed discussion of the properties of such spaces, see Bridson and Haefliger [1] or Burago et al. [3]. It is well known that any complete, simply connected Riemannian manifold having nonpositive sectional curvature is a CAT(0) space. Other examples include the 
classical hyperbolic spaces, Euclidean buildings (see [2]), the complex Hilbert ball with the hyperbolic metric (see [9]; also [14, inequality (4.3)] and subsequent comments), and many others.

\section{Preliminaries}

Let $(X, d)$ be a metric space. A geodesic path joining $x \in X$ to $y \in X$ (or, more briefly, a geodesic from $x$ to $y$ ) is a map $c$ from a closed interval $[0, l] \subset \mathbb{R}$ to $X$ such that $c(0)=x$, $c(l)=y$, and $d\left(c(t), c\left(t^{\prime}\right)\right)=\left|t-t^{\prime}\right|$ for all $t, t^{\prime} \in[0, l]$. In particular, $c$ is an isometry and $d(x, y)=l$. The image $\alpha$ of $c$ is called a geodesic (or metric) segment joining $x$ and $y$. When unique, this geodesic is denoted $[x, y]$. The space $(X, d)$ is said to be a geodesic space if every two points of $X$ are joined by a geodesic, and $X$ is said to be uniquely geodesic if there is exactly one geodesic joining $x$ and $y$ for each $x, y \in X$. A subset $Y \subseteq X$ is said to be convex if $Y$ includes every geodesic segment joining any two of its points.

For complete details and further discussion, see, for example, [1] or [3].

For $\kappa \in(-\infty, 0]$, let $M_{\mathcal{K}}^{2}$ denote the classical surface of curvature $\kappa$. Thus $M_{\kappa}^{2}$ is just the Euclidean plane $\mathbb{E}^{2}$ if $\kappa=0$, and $M_{\kappa}^{2}$ is obtained from the classical hyperbolic plane by multiplying the distance function by $1 / \sqrt{-\kappa}$ if $\kappa<0$.

A geodesic triangle $\Delta\left(x_{1}, x_{2}, x_{3}\right)$ in a geodesic metric space $(X, d)$ consists of three points in $X$ (the vertices of $\Delta$ ) and a geodesic segment between each pair of vertices (the edges of $\Delta)$. A comparison triangle for geodesic triangle $\Delta\left(x_{1}, x_{2}, x_{3}\right)$ in $(X, d)$ is a triangle $\bar{\Delta}\left(x_{1}\right.$, $\left.x_{2}, x_{3}\right):=\Delta\left(\bar{x}_{1}, \bar{x}_{2}, \bar{x}_{3}\right)$ in $M_{\kappa}^{2}$ such that $d_{M_{\kappa}^{2}}\left(\bar{x}_{i}, \bar{x}_{j}\right)=d\left(x_{i}, x_{j}\right)$ for $i, j \in\{1,2,3\}$. Comparison triangles always exist; see [1, Lemma 2.14].

A geodesic metric space is said to be a CAT(0) space if all distances between points on the sides of a geodesic triangle $\Delta$ are no larger than the distances between the corresponding points on any comparison triangle $\bar{\Delta}$ in $\mathbb{E}^{2}$. A geodesic space is said to have Alexandrov curvature bounded below if there exists $\kappa \in(-\infty, 0]$ for which the distances between all points on any geodesic triangle $\Delta$ are no smaller than distances between the corresponding points on any comparison triangle $\bar{\Delta}$ in $M_{\kappa}^{2}$.

Now let $X$ be a CAT(0) space, let $a, x, y \in X$, let $m[x, y]$ denote the midpoint of the segment $[x, y]$ (which is necessarily unique), and let $\varepsilon \in[0,2]$. It is possible to define a modulus $\delta_{a}$ by setting

$$
\delta_{a}(\varepsilon)=\inf \{1-d(a, m[x, y])\}
$$

where the infimum is taken over all points $x, y \in X$ for which $d(a, x) \leq 1, d(a, y) \leq 1$, and $d(x, y) \geq \varepsilon$. By using comparison triangles it is easy to see that $\delta_{a}(\varepsilon) \geq \delta(\varepsilon)$ for all $a \in X$, where $\delta$ is the usual modulus of convexity in $\mathbb{E}^{2}$. In particular, $\delta_{a}(\varepsilon)>0$ if $\varepsilon>0$.

\section{Nearest and farthest points}

In this section we assume that the space $X$ has the geodesic extension property. Although this property is usually defined locally, for a complete CAT $(0)$ space it is equivalent to the assertion that each nonconstant geodesic $c:[a, b] \rightarrow X$ can be extended to a line $c: \mathbb{R} \rightarrow X$. For a technical discussion of this property along with examples, see [1, page 207]. 
Let $C$ be a subset of a CAT(0) space $X$. For $x \in X$, set

$$
\begin{aligned}
& P_{C}(x)=\{y \in C: d(x, y)=\inf \{(d(x, u): u \in C)\}\}, \\
& F_{C}(x)=\{y \in C: d(x, y)=\sup \{(d(x, u): u \in C)\}\} .
\end{aligned}
$$

We now state our main results.

Theorem 3.1. Suppose $X$ is a complete CAT(0) space with the geodesic extension property and with Alexandrov curvature bounded below. Let $C$ be a nonempty closed subset of $X$. Then the set of points of $X$ at which the mapping $P_{C}$ is well defined and single valued is a set of the second Baire category.

Theorem 3.2. Suppose $X$ is a complete CAT(0) space with the geodesic extension property and with Alexandrov curvature bounded below. Let $C$ be a nonempty bounded closed subset of $X$. Then $F_{C}$ is well defined and single valued on a dense subset of $X$.

The proofs of both theorems depend on the fact proved below, which uses the assumption that the curvature is bounded below. This is an analog of a property of uniformly convex Banach space used by Stečkin [16, Lemma 4].

Proposition 3.3. Suppose $X$ is a complete CAT( 0$)$ space satisfying the geodesic extension property and with Alexandrov curvature bounded below by $\kappa \in(-\infty, 0]$. Then if $\varepsilon, d$, and $r$ are fixed positive numbers, there exist $\xi=\xi(\varepsilon, d, r)>0$ and $\xi^{\prime}=\xi^{\prime}(\varepsilon, d, r)>0$ such that if $c, c^{\prime} \in X$ satisfy $d\left(c, c^{\prime}\right)=r$, then

$$
\begin{aligned}
& \operatorname{diam}\left[B(c ; d) \cap\left(X \backslash B\left(c^{\prime} ; d+r-\xi\right)\right)\right]<\varepsilon, \\
& \operatorname{diam}\left[B(c ; d-r+\xi) \cap\left(X \backslash B\left(c^{\prime} ; d\right)\right)\right]<\varepsilon .
\end{aligned}
$$

Proof. We only prove the first conclusion since the formulations are equivalent to one another. If the first conclusion is false then there exist positive numbers $\varepsilon, d, r$, a sequence $\left\{\xi_{i}\right\}$ of positive numbers, and sequences $\left\{c_{i}\right\},\left\{c_{i}^{\prime}\right\}$ in $X$ for which

$$
\operatorname{diam}\left(S_{i}\right) \geq \varepsilon,
$$

where

$$
S_{i}=B\left(c_{i} ; d\right) \cap\left(X \backslash B\left(c_{i}^{\prime} ; d+r-\xi_{i}\right)\right) .
$$

For each $i$ let $u_{i} \in X$ so that $c_{i}$ is on the segment $\left[c_{i}^{\prime}, u_{i}\right]$ and so that $d\left(c_{i}, u_{i}\right)=d$. (Here we are using the geodesic extension property.) Then $u_{i} \in S_{i}$, so by assumption there exists $w_{i} \in S_{i}$ for which $d\left(u_{i}, w_{i}\right) \geq \varepsilon / 2$. Now choose $h_{i}$ on the segment $\left[c_{i}^{\prime}, w_{i}\right]$ so that $d\left(c_{i}^{\prime}, h_{i}\right)=$ $r$. Fix $a, b \in M_{\mathcal{K}}^{2}$ with $d(a, b)=d+r$ and let $\Delta\left(b, \bar{w}_{i}, a\right)$ be the comparison triangle in $M_{\mathcal{K}}^{2}$ corresponding to $\Delta\left(u_{i}, w_{i}, c_{i}^{\prime}\right)$ (with $b$ corresponding to $u_{i}$ and $a$ corresponding to $c_{i}^{\prime}$. See the proof of [1, Lemma 2.14]). The point $\bar{c}_{i}$ of the segment $[a, b]$ corresponding to $c_{i}$ is always the same, so we can label it $c$. Since $d\left(u_{i}, w_{i}\right) \geq \varepsilon / 2$, and since $X$ has Alexandrov curvature bounded below by $\kappa$, it follows that

$$
d\left(h_{i}, c_{i}\right) \geq d\left(\bar{h}_{i}, c\right) .
$$


Since $b, \bar{w}_{i}$ are in the ball $B(a ; d+r)$, with $d\left(b, \bar{w}_{i}\right) \geq \varepsilon / 2$, it follows that $d\left(\bar{h}_{i}, c\right)$ is uniformly bounded away from 0 . Thus there exists $\varepsilon^{\prime} \in(0,2]$ such that $d\left(h_{i}, c_{i}\right) \geq \varepsilon^{\prime} r$. Since

$$
\lim _{i \rightarrow \infty}\left[r+d\left(h_{i}, w_{i}\right)\right]=\lim _{i \rightarrow \infty} d\left(c_{i}^{\prime}, w_{i}\right) \leq r+d,
$$

it follows that $\lim _{i \rightarrow \infty} d\left(h_{i}, w_{i}\right) \leq d$. Also $\lim _{i \rightarrow \infty} d\left(c_{i}, w_{i}\right) \leq d$. Let $z_{i}$ be the midpoint of the segment $\left[h_{i}, c_{i}\right], i=1,2, \ldots$. Since closed balls are convex,

$$
\limsup _{i \rightarrow \infty} d\left(w_{i}, z_{i}\right) \leq d
$$

Since the modulus $\delta:=\delta_{c_{i}^{\prime}}\left(\varepsilon^{\prime}\right)>0$, it is possible to choose $\mu>0$ so small that

$$
(1-\delta) r+d+\mu \leq k<r+d
$$

Also, since $d\left(c_{i}^{\prime}, c_{i}\right)=d\left(c_{i}^{\prime}, h_{i}\right) \equiv r$, it must be the case that

$$
d\left(c_{i}^{\prime}, z_{i}\right) \leq(1-\delta) r
$$

Thus for $i$ sufficiently large,

$$
d\left(c_{i}^{\prime}, w_{i}\right) \leq d\left(c_{i}^{\prime}, z_{i}\right)+d\left(w_{i}, z_{i}\right) \leq(1-\delta) r+d+\mu \leq k .
$$

Since $\lim _{i \rightarrow \infty} d\left(c_{i}^{\prime}, w_{i}\right)=r+d$, we have a contradiction.

For the proof of Theorem 3.1 we introduce some notation. For $x \in X$ and $d=\operatorname{dist}(x$, C) $>0$, put

$$
\begin{gathered}
Y_{\varepsilon}(x)=B(x ; d+\varepsilon) \cap C \quad(\varepsilon>0), \\
d_{\varepsilon}(x)=\operatorname{diam}\left(Y_{\varepsilon}(x)\right), \\
d_{0}(x)=\lim _{\varepsilon \rightarrow 0^{+}} d_{\varepsilon}(x) .
\end{gathered}
$$

Let $\alpha>0$ and denote by $F_{\alpha}=F_{\alpha}(C)$ the set of all points $x \in X$ such that $d_{0}(x) \geq 1 / \alpha$. The proof of the following lemma is a straightforward adaptation of the proof of $[16$, Lemma 2], or the proof of [4, Proposition 2.3]

Lemma 3.4. Let $C$ be a subset of a complete CAT(0) space $X$. Then $F_{\alpha}$ is closed for every $\alpha>0$.

Theorem 3.1 is a direct consequence of the preceding lemma and the following theorem.

Theorem 3.5. Suppose $X$ is a complete CAT(0) space with the geodesic extension property and with Alexandrov curvature bounded below. Let $C$ be a nonempty subset of $X$. Then the set $K(C)$ of all points $x \in X$ for which $d_{0}(x)=0$ is a $G_{\delta}$ set and of the second Baire category. 
Proof (this is a slight modification of Stečkin's original proof [16] in a uniformly convex Banach space setting; see also the proof of [4, Theorem 2.14]). It is clear that

$$
K(C)=X \backslash H(C),
$$

where

$$
H(C)=\bigcup_{n=1}^{\infty} F_{n} .
$$

By Lemma 3.4, $F_{n}$ is closed for all $n$, and we now prove that $F_{n}$ is nowhere dense for all $n$.

Let $x \in F_{n}$ and let $\operatorname{dist}(x, C)=d>0$. Suppose that $N$ is a neighborhood of $x$, and choose $r>0$ so that $B(x ; r) \subset N$. If $\varepsilon \in(0,1 / n)$ is given, then by Proposition 3.3 there exists $\xi_{0}=\xi_{0}(\varepsilon, d, r)>0$ such that if $c, c^{\prime} \in X$ satisfy $d\left(c, c^{\prime}\right)=r$, then

$$
\operatorname{diam}[B(c ; d-r+\xi)] \cap\left(X \backslash B\left(c^{\prime} ; d\right)\right)<\varepsilon<\frac{1}{n}
$$

for all $\xi \in\left(0, \xi_{0}\right]$. Since $\operatorname{dist}(x, C)=d$, there exists $y \in C$ such that $d(x, y)<d+\xi_{0}$. Choose $c \in X$ so that $c \in[x, y]$ and $d(x, c)=r$. Then $c \in N$ and

$$
Y_{\xi_{0}}(c) \subset B\left(c ; d-r+\xi_{0}\right) \cap(X \backslash B(x ; d)) .
$$

This implies that $\operatorname{diam}\left(Y_{\xi_{0}}(c)\right)<1 / n$. Hence $d_{0}(c)<1 / n$ and therefore $c$ is not in $F_{n}$. It follows that $F_{n}$ has empty interior.

Proof of Theorem 3.2. Once Proposition 3.3 has been established, the details of proving that the mapping $F_{C}$ is well defined on a dense subset of $X$ can be found in [10], or deduced by a straightforward modification the Banach space proof given in [11].

To see that $F_{C}$ is single-valued on a dense subset of $X$, suppose $F_{C}(x)$ is nonempty for $x \in X$ and choose $y \in F_{C}(x)$. Now choose $z$ so that $x$ is on the segment $[z, y]$. Choose $u \in C$ with $u \neq y$, and suppose $d(z, u) \geq d(z, y)$. Then

$$
\begin{aligned}
d(z, y) & =d(z, x)+d(x, y) \geq d(z, x)+d(x, u) \\
& \geq d(z, u) \geq d(z, y)
\end{aligned}
$$

Thus

$$
d(z, x)+d(x, u)=d(z, u)
$$

and hence $x$ is on the segment $[z, u]$. Again this contradicts the fact that segments do not bifurcate in $X$. Therefore if $u \in X$ with $u \neq y, d(z, u)<d(z, y)$. It follows that $F_{C}(z)=\{y\}$. Since $z$ can be chosen arbitrarily near $x$, the conclusion follows.

Remark 3.6. One implication of Proposition 3.3 is that in such spaces the intersection of two spheres $S(p ; d) \cap S(q ; r)$ with $d=d(p, q)+r$ consists of exactly one point. This fact and Theorem 3.1 lead to the following result. The proof is an obvious modification of the proof of [13, Theorem 2.2]. 
Theorem 3.7. Suppose $X$ is a complete CAT(0) space with the geodesic extension property and with Alexandrov curvature bounded below. Let $C$ be a closed subset of $X$, suppose $T$ : $C \rightarrow C$ is nonexpansive, and suppose the fixed point set of $T$ has nonempty interior. Then for each $x \in C$ the Picard sequence $\left\{T^{n}(x)\right\}$ converges to a fixed point of $T$.

The following example is relevant to Theorems 3.1 and 3.2.

Definition 3.8. An $\mathbb{R}$-tree is a metric space $T$ such that:

(i) there is a unique geodesic segment (denoted by $[x, y]$ ) joining each pair of points $x, y \in T$

(ii) if $[y, x] \cap[x, z]=\{x\}$, then $[y, x] \cup[x, z]=[y, z]$.

It is known that an $\mathbb{R}$-tree is a CAT(0) space (see [1, page 167]). The following example shows that Theorem 3.2 is false if the assumption that the Alexandrov curvature is bounded below is dropped.

Example 3.9. Consider the complete $\mathbb{R}$-tree $X$ obtained by taking all lines $L_{i}$ in $\ell_{2}$ of the form

$$
L_{i}=\left\{t e_{i}:-\infty<t<\infty\right\},
$$

where $e_{i}$ is the standard $i$ th unit basis vector, and give $X$ the shortest path metric. Let $C_{1}$ be the set

$$
\left\{t e_{i}: t \geq 1+\frac{1}{i}, i=1,2, \ldots\right\}
$$

Then clearly $C_{1}$ is a closed set in $X$ since it is the union of disjoint closed rays with the distance between any two distinct rays at least 2 . However no point in the open set $U:=$ $\{(u, 0,0, \ldots): u<0\}$ has a nearest point in $C_{1}$. Similarly, if $C_{2}$ is the set

$$
\left\{t e_{i}: 0 \leq t \leq 1-\frac{1}{i}, i=1,2, \ldots\right\}
$$

then no point in $U$ has a farthest point in $C_{2}$. Clearly $U$ has nonempty interior.

A metric space is said to be without bifurcating geodesics if any two segments having another common point are either identical, or one contains the other. In view of Example 3.9, the condition that geodesics do not bifurcate is a necessary condition for Theorems 3.1 and 3.2 to hold. Is it sufficient?

Problem 3.10. Can the assumption that the Alexandrov curvature is bounded below in Theorems 3.1 and 3.2 be replaced with the assumption that geodesics do not bifurcate?

We leave another question open. 
Problem 3.11. Do Theorems 3.1 and 3.2 remain true without assuming the geodesic extension property?

\section{Proximity pairs}

We conclude with an observation about set-valued mappings. Here we use $d_{H}$ to denote the usual Hausdorff metric defined on the family of nonempty closed subsets of a metric space $X$.

It is known that if $A$ is a nonempty closed convex subset of a CAT(0) space, then the nearest point projection $P_{A}$ of $X$ onto $A$ is nonexpansive (see [1, page 176]). The following lemma is a straightforward consequence of this fact (see [12]).

Lemma 4.1. Let $A$ be a nonempty closed convex subset of a CAT(0) space $X$. If $C$ and $D$ are nonempty closed and bounded subsets of $X$, then

$$
d_{H}\left(P_{A}(C), P_{A}(D)\right) \leq d_{H}(C, D) .
$$

We will also need the following fact.

Proposition 4.2 [1, page 176]. If $X$ is a CAT(0) space, then the distance function $d$ : $X \times X \rightarrow \mathbb{R}$ is convex.

This means that given any pair of geodesics $c:[0,1] \rightarrow X$ and $c^{\prime}:[0,1] \rightarrow X$ parametrized proportional to arc length, the following inequality holds for all $t \in[0,1]$ :

$$
d\left(c(t), c^{\prime}(t)\right) \leq(1-t) d\left(c(0), c^{\prime}(0)\right)+t d\left(c(1), c^{\prime}(1)\right) .
$$

We now give a consequence of Proposition 4.2. Here

$$
\operatorname{dist}(A, B)=\inf \{d(x, y): x \in A, y \in B\}
$$

The analog of this result holds in reflexive Banach spaces and the proof usually given invokes weak compactness of $A$. Our proof circumvents this approach, using instead the fact that descending sequences of nonempty bounded closed convex sets in a complete CAT(0) space have nonempty intersection (see [1]).

Proposition 4.3. Let $A$ and $B$ be disjoint closed convex subsets of a complete CAT( 0$)$ space $X$ with $A$ bounded. Then the sets

$$
\begin{aligned}
& A_{0}:=\{a \in A: d(a, b)=\operatorname{dist}(a, B) \text { for some } b \in B\}, \\
& B_{0}:=\{b \in B: d(a, b)=\operatorname{dist}(b, A) \text { for some } a \in A\}
\end{aligned}
$$

are nonempty and satisfy

$$
P_{B}\left(A_{0}\right) \subseteq B_{0}, \quad P_{A}\left(B_{0}\right) \subseteq A_{0} .
$$


8 Proximinality in geodesic spaces

Proof. First we observe that if $u, v \in A$ satisfy

$$
\operatorname{dist}(u, B) \leq r, \quad \operatorname{dist}(v, B) \leq r,
$$

then for each $\varepsilon>0$ there exist $w, z \in B$ such that

$$
d(u, w) \leq r+\varepsilon, \quad d(v, z) \leq r+\varepsilon .
$$

Let $t \in(0,1)$ and let $m_{t}$ be the point of the segment $[u, v]$ for which

$$
d\left(u, m_{t}\right)=(1-t) d(u, v), \quad d\left(v, m_{t}\right)=t d(u, v) .
$$

Then if $n_{t}$ is the point of the segment $[w, z]$ for which

$$
d\left(w, n_{t}\right)=(1-t) d(w, z), \quad d\left(z, n_{t}\right)=t d(w, z),
$$

Proposition 4.2 implies $d\left(m_{t}, n_{t}\right) \leq(1-t)(r+\varepsilon)+t(r+\varepsilon)=r+\varepsilon$. Therefore

$$
\operatorname{dist}\left(m_{t}, B\right) \leq r+\varepsilon
$$

and since $\varepsilon>0$ is arbitrary, $\operatorname{dist}\left(m_{t}, B\right) \leq r$. This proves that the set

$$
\{x \in A: \operatorname{dist}(x, B) \leq r\}
$$

is convex.

Now let $d=\operatorname{dist}(A, B)$ and for each $n=1,2, \ldots$, let

$$
A_{n}=\left\{x \in A: \operatorname{dist}(x, B) \leq d+\frac{1}{n}\right\} .
$$

Then $\left\{A_{n}\right\}$ is a sequence of closed convex subsets of $A$, so $\bigcap_{n=1}^{\infty} A_{n}$ is nonempty, closed, and convex. Moreover, if $u \in \bigcap_{n=1}^{\infty} A_{n}$, then there exists $w \in B$ (since $B$ is proximinal) such that $d(u, w)=\operatorname{dist}(u, B)=\operatorname{dist}(A, B)$. This proves that $A_{0}$ and $B_{0}$ are nonempty. If $y \in$ $P_{B}\left(A_{0}\right)$, then $y=P_{B}(x)$ for some $x \in A_{0}$, so $d(x, y)=\operatorname{dist}(A, B)$. Hence $y \in B_{0}$. Similarly, $P_{A}\left(B_{0}\right) \subseteq A_{0}$.

Theorem 4.4. Suppose $(X, d)$ is a complete CAT(0) space, let $K$ be a bounded closed convex subset of $X$, and suppose $T$ is a nonexpansive set-valued mapping of $K$ into $2^{K}$ whose values are nonempty compact subsets of $K$. The $T$ has a fixed point.

Proof. Since asymptotic centers of bounded sequences are unique in CAT(0) spaces, it is possible to follow standard proof of the analog of the theorem in uniformly convex Banach spaces (cf., [8, page 165]). (For extensions of this result, see $[5,6]$.)

Given the preceding facts, the following result can be obtained by repeating the proof of [12, Theorem 3.3], changing the norm to distance, and applying Theorem 4.4 instead of Lim's theorem at the relevant step. 
Theorem 4.5. Let $X$ be a complete CAT(0) space and let $A, B$ be nonempty closed convex subsets of $X$ with $A$ bounded. Let $T: A \rightarrow 2^{B}$ be a multivalued mapping such that

(i) for each $x \in A, T(x)$ is a nonempty compact subset of $B$,

(ii) $d_{H}(T(x), T(y)) \leq d(x, y)$ for all $x, y \in A$,

(iii) $T\left(A_{0}\right) \subseteq B_{0}$.

Then there exists $x_{0} \in A$ such that

$$
\operatorname{dist}\left(x_{0}, T\left(x_{0}\right)\right)=\operatorname{dist}(A, B)=\inf \{\operatorname{dist}(x, T(x)): x \in A\} .
$$

\section{Acknowledgments}

We are deeply indebted to Stefan Cobzaş for providing us with his English translation of Stečkin's paper [16]. The first author was supported by Thailand Research Fund under Grant BRG4780013, and by the Royal Golden Jubilee program under Grant PHD/0250/ 2545. This work was carried out while she was visiting the University of Iowa. She expresses her gratitude to the Department of Mathematics.

\section{References}

[1] M. R. Bridson and A. Haefliger, Metric Spaces of Non-Positive Curvature, Fundamental Principles of Mathematical Sciences, vol. 319, Springer, Berlin, 1999.

[2] K. S. Brown, Buildings, Springer, New York, 1989.

[3] D. Burago, Y. Burago, and S. Ivanov, A Course in Metric Geometry, Graduate Studies in Mathematics, vol. 33, American Mathematical Society, Rhode Island, 2001.

[4] S. Cobzaş, Geometric properties of Banach spaces and the existence of nearest and farthest points, Abstract and Applied Analysis 2005 (2005), no. 3, 259-285, Proceedings of the International Workshop on Small Sets in Analysis (Technion - Israel Institute of Technology) (E. Matoušková, S. Reich and A. J. Zaslavski, eds.), Hindawi, New York, June 2003, pp. 259-285.

[5] S. Dhompongsa, A. Kaewkhao, and B. Panyanak, Lim's theorems for multivalued mappings in CAT(0) spaces, Journal of Mathematical Analysis and Applications 312 (2005), no. 2, 478-487.

[6] S. Dhompongsa, W. A. Kirk, and B. Panayak, Nonexpansive set-valued mappings in metric and Banach spaces, to appear in Journal of Nonlinear and Convex Analysis.

[7] M. Edelstein, On nearest points of sets in uniformly convex Banach spaces, Journal of the London Mathematical Society. Second Series 43 (1968), 375-377.

[8] K. Goebel and W. A. Kirk, Topics in Metric Fixed Point Theory, Cambridge Studies in Advanced Mathematics, vol. 28, Cambridge University Press, Cambridge, 1990.

[9] K. Goebel and S. Reich, Uniform Convexity, Hyperbolic Geometry, and Nonexpansive Mappings, Monographs and Textbooks in Pure and Applied Mathematics, vol. 83, Marcel Dekker, New York, 1984.

[10] W. A. Kirk, Nearest and farthest points of closed sets in hyperbolic spaces, Constantin Carathéodory: An International Tribute, Vol. I, II (Th. M. Rassias, ed.), World Scientific, New Jersey, 1991, pp. 581-591.

[11] _ Property $(\beta)$ and Edelstein's algorithms for constructing nearest and farthest points, Banach Spaces (Mérida, 1992) (B. L. Lin, ed.), Contemporary Math., vol. 144, American Mathematical Society, Rhode Island, 1993, pp. 149-158.

[12] W. A. Kirk, S. Reich, and P. Veeramani, Proximinal retracts and best proximity pair theorems, Numerical Functional Analysis and Optimization 24 (2003), no. 7-8, 851-862.

[13] W. A. Kirk and B. Sims, Convergence of Picard iterates of nonexpansive mappings, Polish Academy of Sciences. Bulletin. Mathematics 47 (1999), no. 2, 147-155. 


\section{Proximinality in geodesic spaces}

[14] S. Reich and I. Shafrir, Nonexpansive iterations in hyperbolic spaces, Nonlinear Analysis 15 (1990), no. $6,537-558$.

[15] S. Reich and A. J. Zaslavski, Well-posedness and porosity in best approximation problems, Topological Methods in Nonlinear Analysis 18 (2001), no. 2, 395-408.

[16] S. B. Stečkin, Approximation properties of sets in normed linear spaces, Revue Roumaine de Mathématiques Pures et Appliquées 8 (1963), 5-18 (Russian).

[17] T. Zamfirescu, Extending Stechkin's theorem and beyond, Abstract and Applied Analysis 2005 (2005), no. 3, 255-258, Proceedings of the International Workshop on Small Sets in Analysis (Technion - Israel Institute of Technology) (E. Matoušková, S. Reich and A. J. Zaslavski, eds.), Hindawi, New York, June 2003, pp. 255-258.

A. Kaewcharoen: Department of Mathematics, Faculty of Science, Naresuan University, Phitsanulok 65000, Thailand

E-mail address: anchaleeka@nu.ac.th

W. A. Kirk: Department of Mathematics, University of Iowa, Iowa City, IA 52242-1419, USA

E-mail address: kirk@math.uiowa.edu 


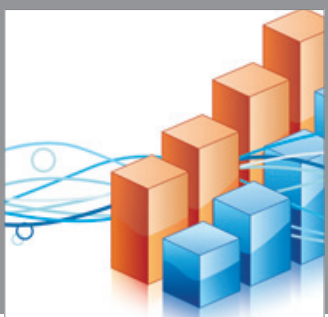

Advances in

Operations Research

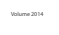

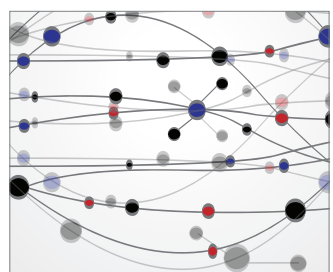

\section{The Scientific} World Journal
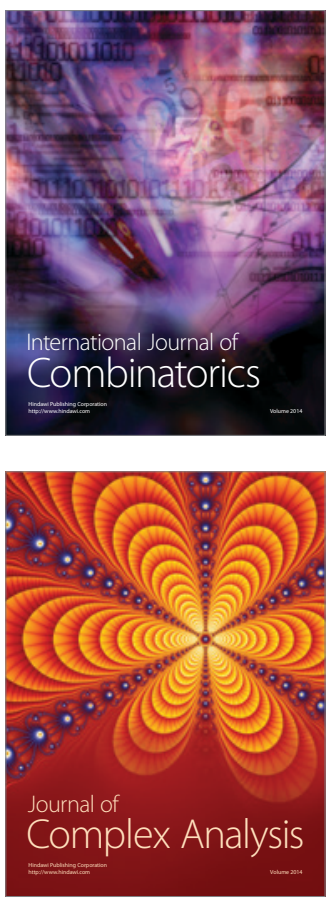

International Journal of

Mathematics and

Mathematical

Sciences
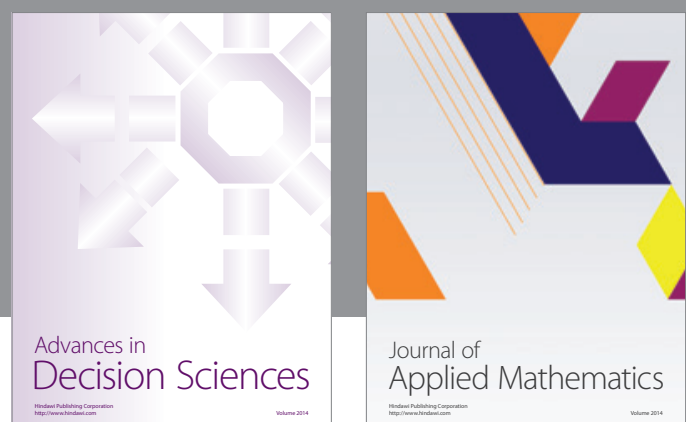

Journal of

Applied Mathematics
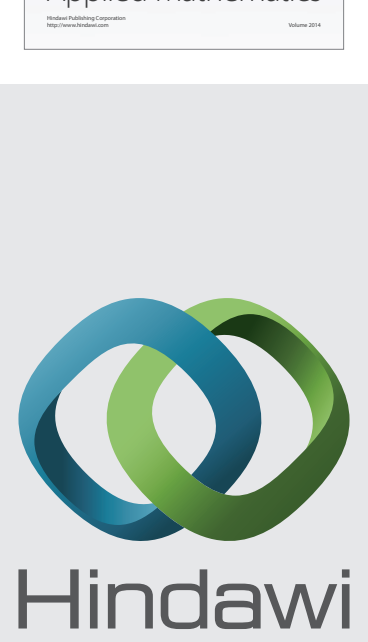

Submit your manuscripts at http://www.hindawi.com
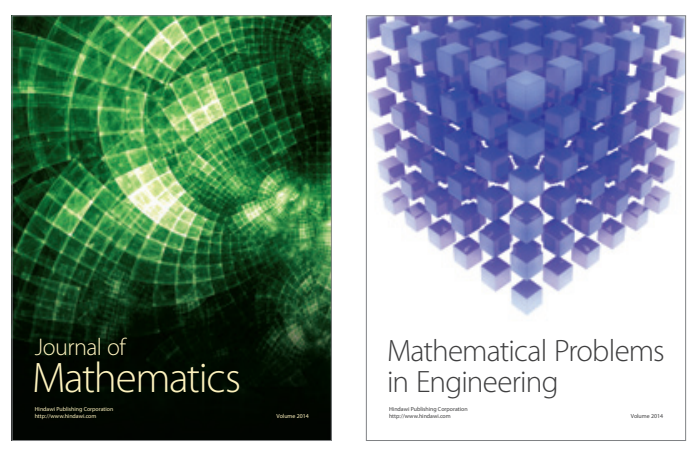

Mathematical Problems in Engineering
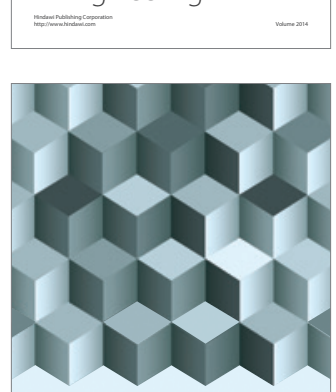

Journal of

Function Spaces
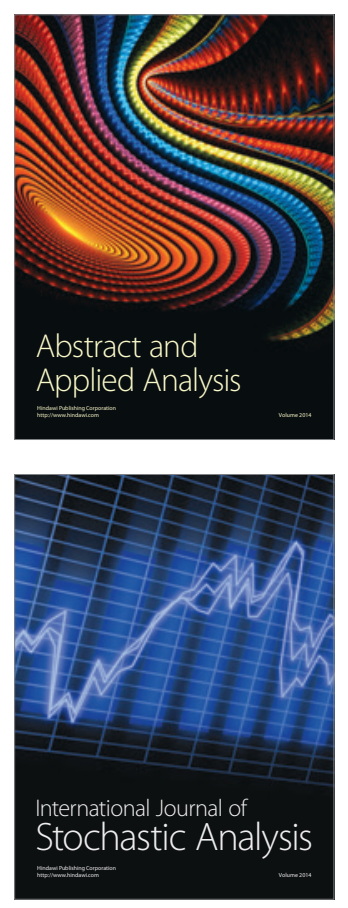

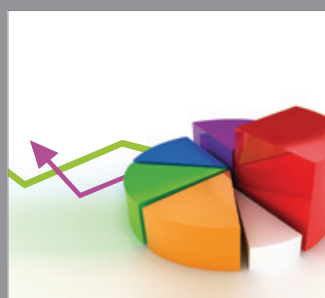

ournal of

Probability and Statistics

Promensencen
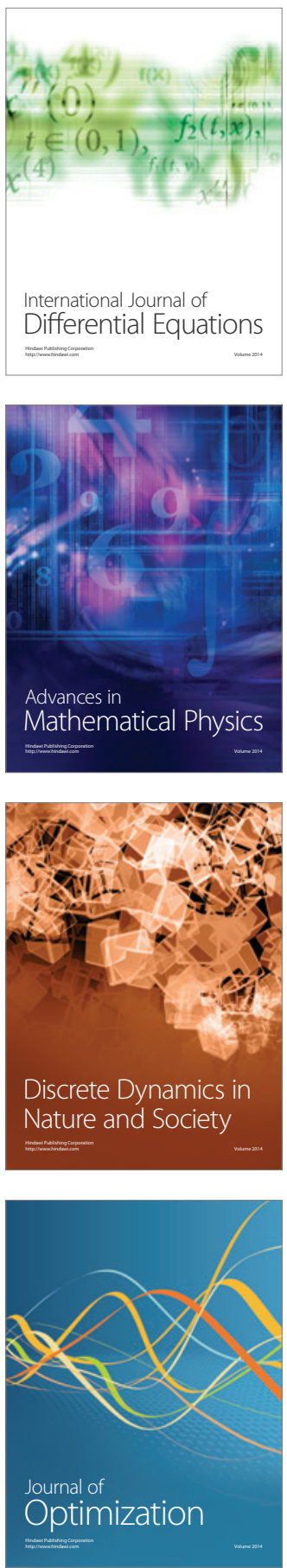